A

Centromere 1

Centromere 2

Centromere 3

Figure S2

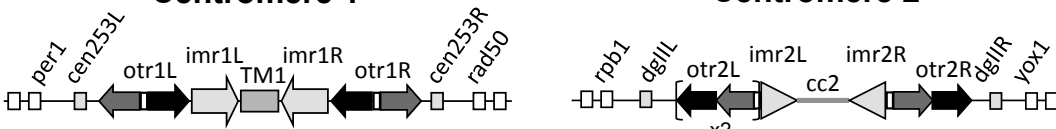
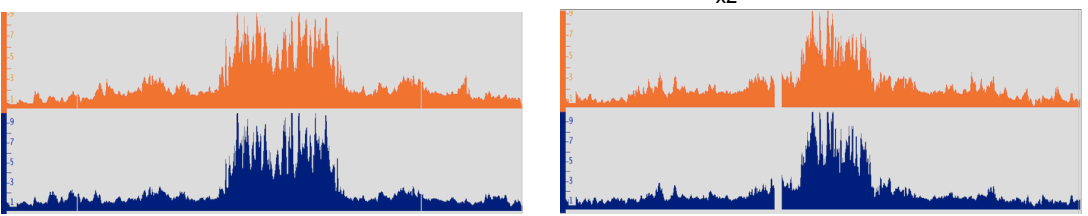

Telomere 1L

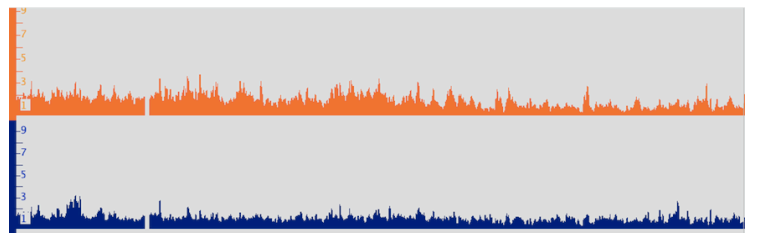

Telomere 2L

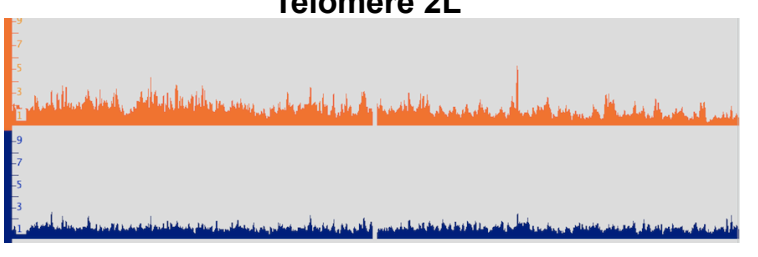

rDNA 3L
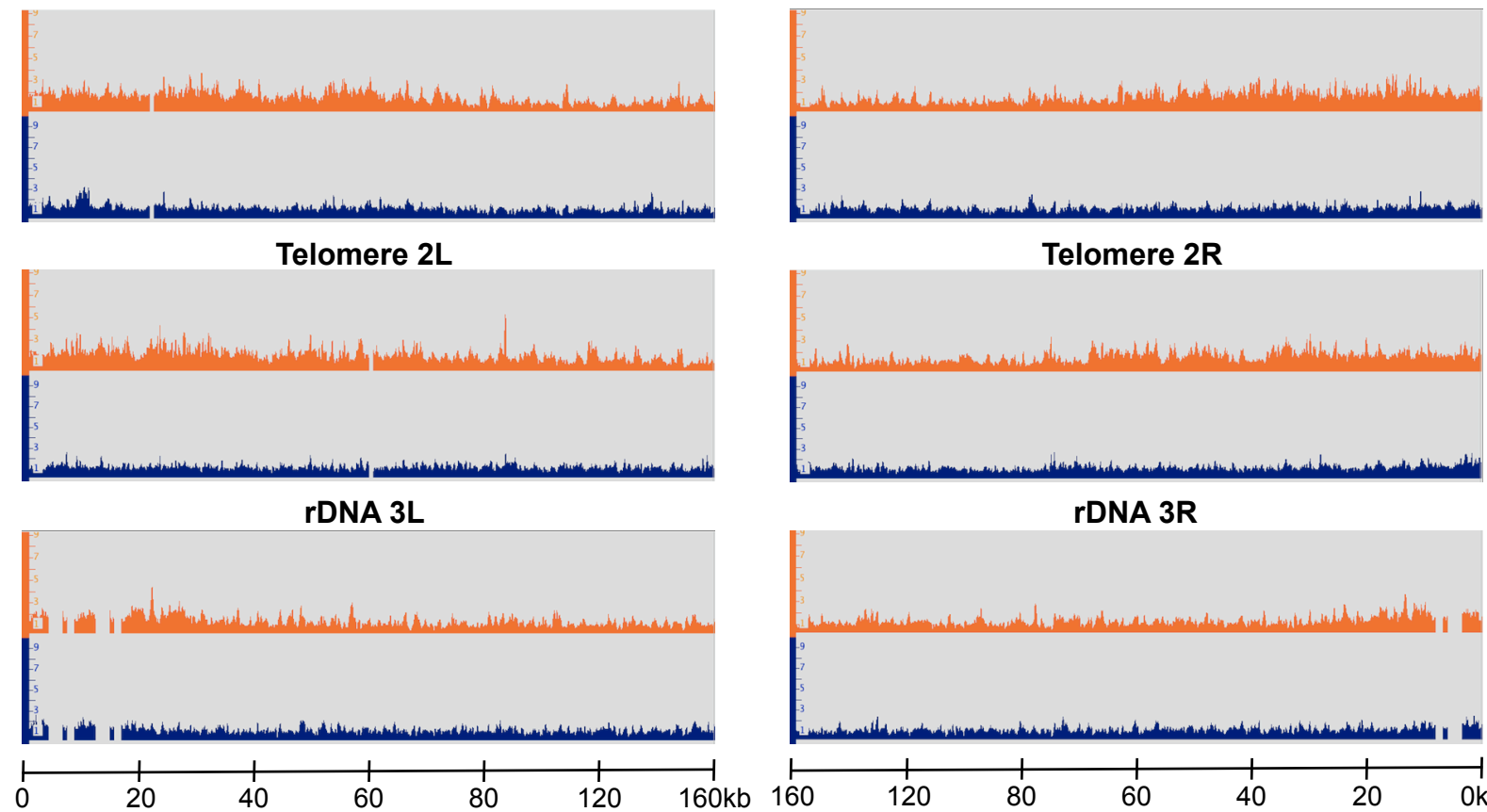

Telomere 2R

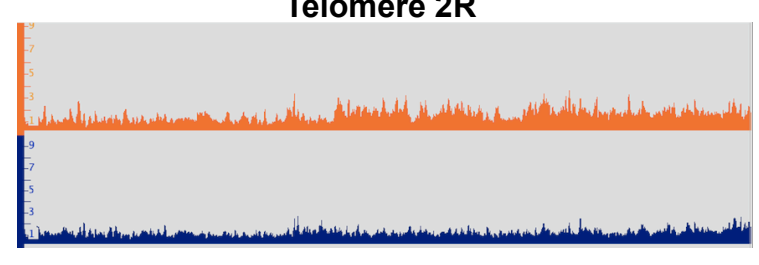

rDNA 3R

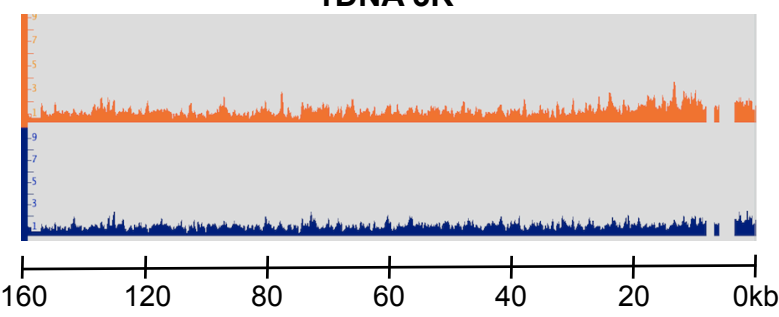

Endog. CENP-A ${ }^{\text {Cnp1 }}$ nmt81-CENP-A

B

$\frac{\text { Endg }}{\mathrm{T} \mathrm{IP}} \frac{\mathrm{nm} 81}{\mathrm{~T} \mathrm{IP}}$
---- otr
--- act

C

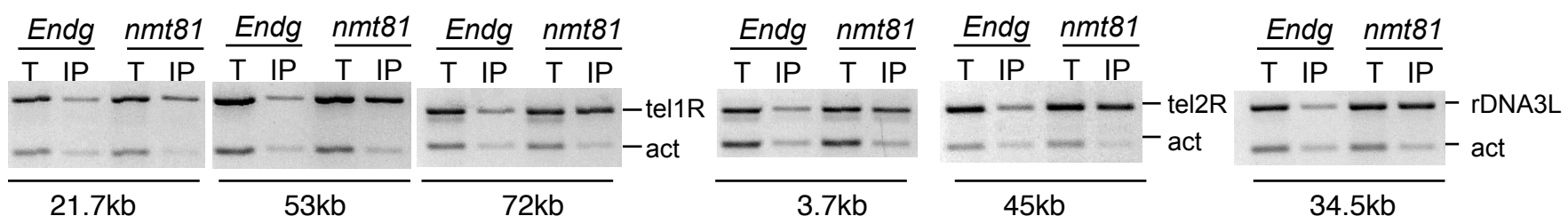

D

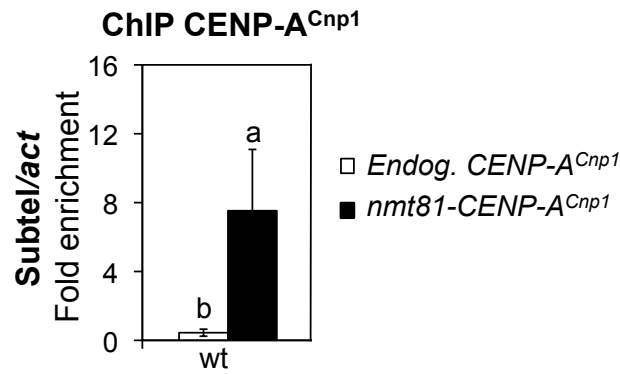




\section{Figure S2}

Figure S2. Additional CENP-A ${ }^{C n p 1}$ accumulates at subtelomeric and pericentromeric regions.

(A) Genome browser view showing ChIP- chip occupancy profiles for wild-type (Endg.) levels in blue or additional CENP-A ${ }^{\text {Cnp1 }}$ levels in orange ( $\left.n m t 81-C E N P-A^{C n p 1}\right)$ at centromeres, pericentromeric outer repeats ( $\mathrm{x} 2, \mathrm{x} 4, \mathrm{x} 9$ indicate the number of repetitive elements) or subtelomeric / rDNA-adjacent regions. Data on the $\mathrm{Y}$ axis are presented in linear scale and $X$-axis shows genome positions (distance from the chromosome end at telomeres and rDNA loci is indicated in kb). ChIP-PCR analysis of CENP-ACnp1 association with pericentromeric outer repeats (B) or subtelomeric / rDNA-adjacent regions (C) expressing wild-type (Endog.) or additional (nmt81) levels of CENP-A ${ }^{C n p 1}$. Enrichment of pericentromeric outer repeats (A, otr primers), telomeric $(B$, primers reside $21.7 \mathrm{~kb}, 53 \mathrm{~kb}$ and $72 \mathrm{~kb}$ from the left telomere of chromosome $\mathrm{I}$ and $3.7 \mathrm{~kb}$ and $47.9 \mathrm{~kb}$ from the right end of chromosome II) )and rDNA adjacent regions (primers reside $34.5 \mathrm{~kb}$ from the rDNA repeats on left arm of chromosome III) was assessed compared to actin (act) in immunoprecipitated (IP) relative to total (T) extract. (D) ChIP-qPCR analysis of CENP-A ${ }^{C n p 1}$ association with a subtelomeric region from the right telomere of chromosome I ( $53 \mathrm{~kb}$ from the end) expressing wild-type (Endog.) or additional (nmt81-CENP-A ${ }^{C n p 1}$ ) levels of CENP-ACnp1. Enrichment on the sub-telomeric region was normalized to the signal obtained for the euchromatic gene encoding actin (act1+). Error bars indicate S.D. from 3 biological replicates. Mean values marked with different letter $(a$ or $b$ ) indicate results significantly different from each other, as established by One Way ANOVA and Holm-Sidak test for multiple comparison $(P<0.01)$. 